\title{
Determinación de indicadores de eutrofización en el embalse Río Tercero, Córdoba (Argentina) ${ }^{1}$
}

\author{
Determination of trophic indicators in Rio Tercero reservoir, Cordoba (Argentina)
}

\author{
Claudia Ledesma ${ }^{2 *}$, Matias Bonansea ${ }^{3}$, Claudia Maria Rodriguez ${ }^{3}$ e Angel Ramon Sánchez Delgado ${ }^{4}$
}

RESUMEN - La eutrofización es una de las problemáticas ambientales más importantes de lagos y embalses. El embalse Río Tercero (Argentina) se encuentra afectado por diversas actividades antrópicas que impactan negativamente sobre el recurso. A la hora de generar un programa de monitoreo de los sistemas acuáticos, una de las consideraciones más importante es poder definir las variables más significativas. El análisis multivariado se presenta como una herramienta alternativa. El objetivo de este trabajo fue determinar la calidad del agua y estado trófico del embalse Río Tercero, con el fin de generar bases para una adecuada gestión de los recursos hídricos. Se realizaron campañas de muestreos durante los años 2003 a 2006 donde se midieron parámetros de calidad del agua. Se determino el estado trófico del reservorio. Se realizó un análisis multivariado, generando una matriz de correlación y un análisis de componentes principales (ACP). El embalse experimentó un deterioro en su calidad, pasando desde un estado de mesotrófico a eutrófico. Se demostró que existe correlación positiva entre la clorofila- a y las variables fósforo total $(r=0.83)$, oxigeno disuelto $(r=0.51)$ y temperatura $(r=0.43)$. El ACP explicó el $65,6 \%$ de la variabilidad total de los datos. El análisis multivariado estableció que las variables más significativas para explicar la variabilidad en el reservorio fueron clorofila-a, fósforo total y temperatura, lo que demuestra que los florecimientos algales deberían ocurrir en las estaciones primavera-verano. Los resultados obtenidos permiten generar un programa preliminar de monitoreo y gestión extrapolable a otros recursos acuáticos.

Palabras claves: Calidad del agua. Eutrofización. Análisis de componentes principales. Análisis de correlación.

\begin{abstract}
Eutrophication is one of the most important environmental problems in lakes and reservoirs. Río Tercero reservoir (Argentina) is affected by various human activities that impact negatively on the system. When generating a monitoring program of aquatic systems, one of the most important considerations is to define the most significant variables. Multivariate analysis was presented as an alternative tool. The aim of this study was to determine water quality and trophic status in Río Tercero reservoir, in order to generate an adequate basis for management of water resources. Sampling campaigns were conducted during the years 2003 to 2006. Water quality parameters were measured. We determined the trophic status of the reservoir. Multivariate analysis was performed, generating a correlation matrix and a principal component analysis (PCA). The reservoir experienced a deterioration in water quality, from mesotrophic to eutrophic state. It was shown that there is positive correlation between chlorophyll-a and total phosphorus $(r=0.83)$, dissolved oxygen $(r=0.51)$ and temperature $(r=0.43)$. The PCA explained the $65.6 \%$ of the total variability of the data. Multivariate analysis established that the most significant variables were chlorophyll-a, total phosphorus and temperature, demonstrating that algal blooms should occur in spring and summer seasons. The results obtained allow to generate a preliminary program of monitoring and management applied to other aquatic resources.
\end{abstract}

Key words: Water quality. Eutophication. Principal components analysis. Correlation analysis.

\footnotetext{
*Autor para correspondência

${ }^{1}$ Recebido para publicação em 20/11/2011; aprovado em 29/01/2013

Parte da Dissertação de Grau do segundo autor apresentada ao Curso de Graduação em Biologia da Universidade Nacional de Rio Cuarto

${ }^{2}$ Departamento de Estudios Básicos y Agropecuarios/Faculdade Agronomía y Veterinaria, Universidad Nacional de Río Cuarto, Ruta Nacional n. 36 , Km, 601, (5800) Río Cuarto, Córdoba, Argentina, cledesma@ayv.unrc.edu.ar

${ }_{3}^{3}$ Departamento de Estudios Básicos y Agropecuarios/Becario CONICET, Universidad Nacional de Río Cuarto, Fac. Agronomía y Veterinaria, Ruta Nacional n. 36, Km. 601, (5800) Río Cuarto, Córdoba, Argentina, mbonansea@ayv.unrc.edu.ar; m_c_rodriguez@yahoo.com

${ }^{4}$ Departamento Matemática, Universidade Federal Rural do Rio de Janeiro, ICE, BR 465, Km 7, seropédica, Rio de Janeiro-RJ, Brasil, 23.851-000, asanchez@ufrrj.br
} 


\section{INTRODUCCIÓN}

Los embalses son reservorios de agua utilizados para satisfacer las necesidades de la sociedad y medio ambiente. Entre las más destacadas figuran la generación de energía, la provisión de agua para consumo humano, animal y riego, la atenuación de crecientes, la recarga de napas subterráneas, la provisión de hábitat para una gran cantidad de especies animales y vegetales (SRIWONGSITANON et al., 2011). Entre los problemas ambientales de lagos y embalses, la eutrofización es uno de los más frecuentes. Este fenómeno que se define como un proceso de deterioro de la calidad del recurso, se origina por el enriquecimiento de nutrientes, principalmente nitrógeno y fósforo, condicionando la utilización de los mismos y ejerciendo grandes impactos ecológicos, sanitarios y económicos a escala regional (GIRÃ̃ et al., 2007; STEFOULI et al., 2005).

Con respecto a los ambientes acuáticos del centro de Argentina, el estado trófico de los mismos, varía entre mesotróficos y eutróficos, dependiendo de la estación del año (AMÉ et al., 2003; BAZÁN et al., 2005; BOLTOVSKOY; FOGGETTA, 1985; MARIAZZI; ROMERO, 1983; RODRIGUEZ et al., 1997). El embalse Río Tercero, se encuentra altamente influenciado por la agricultura, el manejo pecuario en alta pendiente, la erosión del suelo, la actividad minera, la descarga de efluentes domésticos no tratados y los provenientes de industrias. Como resultado, la carga de materia orgánica es generalmente alta, lo que provoca una desorganización en el normal funcionamiento del ecosistema y genera la estimulación de una serie de cambios sintomáticos, tales como, pérdida de los usos potenciales del agua, color, olor y sabor desagradable, trastornos en la salud humana y animal, disminución de la biodiversidad, reducción de la penetración de la luz, mortandad de peces, pérdidas económicas, aumentos en las poblaciones de microorganismos patógenos y vectores de enfermedades, informándose en varias ocasiones importantes florecimientos o blooms de cianobacterias potencialmente tóxicas para la salud humana y animal (BONANSEA, 2007; MANCINI et al., 2008; MANCINI et al., 2010; PIEROTTO et al., 2007; RODRIGUEZ et al., 2007).

Recientemente se han publicado artículos científicos que tratan de generar un programa de monitoreo y gestión de los recursos acuáticos (BARROS et al., 2010, ANDRADE et al., 2010, DO VASCO et al., 2011). En todos los casos lo más importante es poder definir las variables más significativas para caracterizar el problema. El análisis multivariado es una técnica de utilidad ya que permite la reducción de dimensión, pudiendo describir y analizar observaciones multidimensionales y así comprender la relación entre variables evaluadas simultáneamente sobre una misma unidad, como también comparaciones, agrupaciones y/o clasificaciones de observaciones (BARROS ALEXANDRE et al., 2010).

El objetivo de este trabajo fue determinar la calidad del agua y estado trófico del embalse Río Tercero (Argentina) utilizando técnicas de análisis estadístico multivariado, con el fin de generar bases para una adecuada gestión de los recursos hídricos de la región.

\section{MATERIAL Y MÉTODOS}

El embalse Río Tercero se encuentra en el valle de Calamuchita en la región de las Sierras Chicas de la provincia de Córdoba (Argentina) $\left(64^{\circ} 23^{\prime} \mathrm{O}\right.$ y $\left.32^{\circ} 10^{\prime} \mathrm{S}\right)$ y a $529.4 \mathrm{~m}$ del nivel del mar. Este reservorio representa el cuerpo de agua artificial más grande de Córdoba con una superficie de 4.600 ha, una profundidad media de $12 \mathrm{~m}$, una máxima de $46 \mathrm{~m}$ y un tiempo de residencia de 0,84 años (BOLTOVSKOY; FOGETTA, 1985). Tiene como tributarios a los ríos Santa Rosa, Amboy, Grande, Quillinzo y La Cruz, y como único efluente al río Tercero (Figura 1). En el año 1983, comenzó a funcionar en la costa sur del lago una central termonuclear, la cual utiliza las aguas del embalse con fines de enfriamiento de su reactor nuclear (MARIAZZI et al., 1992).

Figura 1 - Ubicación de los sitios de muestreo en el embalse Río Tercero y sus tributarios

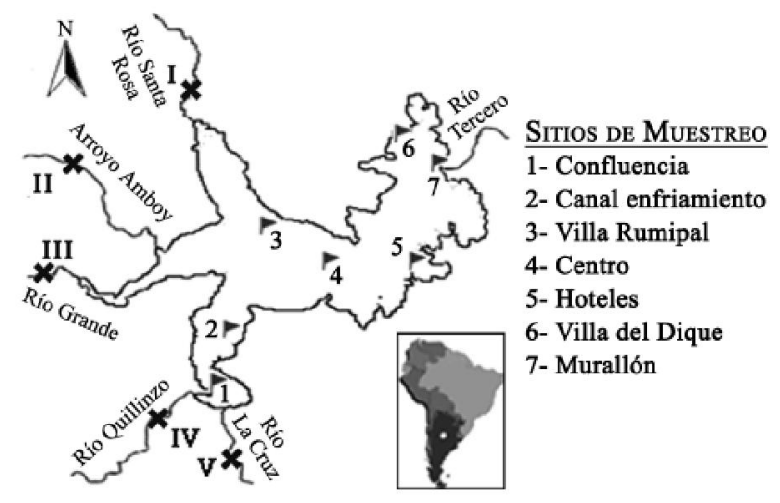

Los muestreos fueron realizados durante el período noviembre de 2003 a diciembre de 2006, con un total de 19 campañas. Las muestras fueron obtenidas a $20 \mathrm{~cm}$ de profundidad o zona eufótica. Los parámetros estudiados fueron: temperatura del agua (T), $\mathrm{pH}$, oxígeno disuelto (OD) y transparencia utilizando la profundidad del disco de Secchi (DS), nitrógeno total (NT), fósforo 
total (PT) y clorofila-a (Cl-a). La metodología de toma de muestras, almacenamiento, conservación y técnicas analíticas se realizaron según APHA (2000).

Para la clasificación del estado trófico se aplicó el Programa Internacional para la Organización, Cooperación y Desarrollo Económico de aguas interiores (OECD, 1982), el cuál proporciona los valores límites de PT, Cl-a y DS, en el ámbito de los lagos de zonas templadas (Tabla 1).

Para identificar las variables que determinaron la variabilidad en la calidad del agua en el embalse Río Tercero, se realizó un análisis estadístico descriptivo multivariado, utilizando el software InfoStat Professional (DI RIENZO et al., 2011). Se elaboró una matriz de correlación para encontrar la co-variación de cada par de variables medidas. En este caso, el coeficiente de correlación muestral representó la covarianza de los valores muestrales estandarizados, asumiendo valores en el intervalo [-1;1], donde el signo indicó la dirección de la asociación. Se utilizó el coeficiente de correlación de Pearson, el cual es una medida de la magnitud de la asociación lineal entre dos variables que no depende de las unidades de medida de las variables originales. Para las variables j-ésima y k-ésima se define como:

$r_{j k}=\frac{S_{j k}}{\sqrt{S_{j}^{2} S_{k}^{2}}}=\frac{\left(\sum_{i=l}^{n}\left(x_{i j}-x_{j}\right)\left(x_{i k}-x_{j}\right) /(n-1)\right)}{\left(\left(\sum_{i=l}^{n}\left(x_{i j}-x_{j}\right)^{2}\right) /(n-1)\right)\left(\left(\sum_{i=l}^{n}\left(x_{i k}-x_{k}\right)^{2}\right) /(n-1)\right)}$

donde $S_{j k}$ es la covarianza entre la variable $j$ y la variable $k, S_{j}^{2}{ }_{j} S^{2}{ }_{k}$ son las varianzas de las variables $j \mathrm{y}$ $k$ respectivamente (DI RIENZO et al., 2011).

Por medio del análisis de componentes principales (ACP), se analizó la variabilidad total de la calidad del agua y se asociaron las variables temporalmente (BALZARINI et al., 2008). Además, con el ACP se identificaron tendencias generales y relaciones entre las variables, por medio de la construcción de ejes artificiales que permitieron proyectar las observaciones y las variables simultáneamente en un mismo plano.

\section{RESULTADOS Y DISCUSIÓN}

La ubicación geográfica y el origen de los reservorios, conjuntamente con la calidad del suelo que drenan y los afluentes que reciben, determinan los parámetros físicos, químicos y biológicos y por ende la productividad de los mismos.

Considerando que el proceso de eutrofización es un fenómeno afectado por un número variable de factores físicos, químicos, biológicos y sociales (ROCHA et al., 2010; SILVA et al., 2006), durante el período en estudio, el embalse Río Tercero se encontraría experimentando un deterioro en la calidad del agua y un avance progresivo hacia un estado elevado de eutrofia, pasando desde mesotrófico a eutrófico (Tabla 2).

En el año 2003, se observó que la condición del embalse estacionalmente fue de caracterísiticas mesotrófícas. Durante el año 2004, esta situación presentó una tendencia al eutrofismo, mientras que en los años 2005 y 2006 la condición fue claramente eutrófica. Esta situación fue similar a la demostrada por BAZAN et al. (2005) y PIEROTTO et al. (2007) en otros reservorios del centro del país.

Para obtener la covariancia de cada par de variables medidas sobre cada una de las unidades experimentales, se generó una matriz de correlación como se muestra en la Tabla 3. HELENA et al. (2000), considera como asociaciones significativas aquellas variables con coeficientes de correlación $r>0.5$. Este mismo criterio fue adoptado en este estudio.

La determinación de la $\mathrm{Cl}$-a, expresada como pigmentos fotosintéticos por unidad de volumen, en un reservorio acuático, es fundamental para evaluar su condición trófica (RYDING; RAST, 1992). Se observó una alta correlación positiva entre las variables Cl-a y PT $(\mathrm{r}=0.83)$. Este mismo tipo de correlación fue encontrado por Boltovskoy; Foggetta (1983) y Mariazzi et al. (1992), al estudiar este mismo reservorio. Durante el período analizado se determinó un incremento en la concentración media de estas dos variables. Esta asociación responde a lo esperado, ya que un aumento en la concentración de nutrientes, principalmente PT, puede producir aumentos

Tabla 1- Valores límites de la OECD para clasificación trófica. PT Medio: Media de la concentración de PT de un lago; Cl-a Media: Media de la concentración de clorofila-a en aguas superficiales; Cl-a Máx.: Pico de la concentración de clorofila-a en aguas superficiales; DS Medio: Media de la transparencia del disco de Secchi; DS Mín.: Mínimo de la transparencia del disco de Secchi (OECD, 1982)

\begin{tabular}{lccccc}
\hline \multicolumn{1}{c}{ Categoría Trófica } & PT Medio $(\mu \mathrm{g} / \mathrm{L})$ & Cl-a Media $(\mu \mathrm{g} / \mathrm{L})$ & Cl-a Máx. $(\mu \mathrm{g} / \mathrm{L})$ & DS Medio $(\mathrm{m})$ & DS Mín. $(\mathrm{m})$ \\
\hline Ultraolitrófico & $<4$ & $<1$ & $<2,5$ & $>12$ & $>6$ \\
Oligotrófico & $<10$ & $<2,5$ & $<8$ & $>6$ & $>3$ \\
Mesotrófico & $10-35$ & $2,5-8$ & $8-25$ & $6-3$ & $3-1,5$ \\
Eutrófico & $35-100$ & $8-25$ & $25-75$ & $3-1,5$ & $1,5-0,7$ \\
Hipereutrófico & $>100$ & $>25$ & $>75$ & $<1,5$ & $<0,7$ \\
\hline
\end{tabular}


Tabla 2 - Parámetros de calidad del agua evaluados durante los años 2003-2006

\begin{tabular}{lcrrrr}
\hline \multirow{2}{*}{ Parámetro } & \multirow{2}{*}{ Unidad } & \multicolumn{4}{c}{ Año de estudio } \\
\cline { 3 - 6 } & & 2003 & 2004 & 2005 & 2006 \\
\hline PT Medio & $(\mu \mathrm{g} / \mathrm{L})$ & 20,0 & 23,0 & 41,0 & 40,0 \\
Cl-a Media & $(\mu \mathrm{g} / \mathrm{L})$ & 4,5 & 6,4 & 10,3 & 10,4 \\
Cl-a Máx. & $(\mu \mathrm{g} / \mathrm{L})$ & 8,0 & 14,0 & 15,0 & 15,5 \\
DS Medio & $(\mathrm{m})$ & 2,3 & 2,4 & 2,9 & 2,6 \\
DS Mín. & $(\mathrm{m})$ & 1,3 & 0,6 & 1,4 & 0,7 \\
\hline
\end{tabular}

Tabla 3 - Matriz de correlación para las variables analizadas. Por debajo de la diagonal principal se observan los coeficientes de correlación r. Solo se muestran como super-índices las probabilidades asociadas a la prueba de hipótesis de correlación nula para las variables con coeficientes de correlación considerables

\begin{tabular}{lccccccc}
\hline & $\mathrm{T}$ & $\mathrm{pH}$ & $\mathrm{OD}$ & $\mathrm{DS}$ & $\mathrm{Cl}-\mathrm{a}$ & $\mathrm{PT}$ & $\mathrm{NT}$ \\
\hline $\mathrm{T}$ & & & & & & & \\
PH & -0.180 .12 & & & & & & \\
OD & -0.13 & -0.07 & & & & & \\
DS & -0.20 & 0.01 & -0.02 & & & & \\
Cl-a & $0.43<0.001$ & -0.06 & $0.51<0.001$ & -0.04 & & & \\
PT & 0.32 & -0.17 & -0.34 & -0.02 & $0.83<0.001$ & & \\
NT & 0.29 & 0.03 & -0.06 & 0.07 & 0.26 & 0.27 & \\
\hline
\end{tabular}

en la aparición de florecimientos o altos niveles de Cl-a (RYDING and RAST, 1992). El aumento en la concentración de PT, puede estar relacionado con la intensificación de las actividades agricola-ganaderas desarrolladas en la cuenca del reservorio, tal como lo hallado por BAZAN et al. (2005). Además un incremento de esta variable puede deberse a la descarga, vía sistemas de drenajes, de las poblaciones ubicadas en el perilago o por escorrentías.

A diferencia de otros autores, si bien se debería haber encontrado una correlación positiva significativa entre Cl-a y NT (RODRIGUEZ et al., 1997), macronutriente más importante luego del PT, esto no pudo demostrarse durante el período estudiado.

La. Si bien el coeficiente de correlación entre la Cl-a y $\mathrm{T}$ fue de moderada a baja $(\mathrm{r}=0.43)$, la $\mathrm{T}$ es uno de los parámetros físicos más importantes en el estudio de la calidad del agua de un reservorio. Con respecto a la eutrofización, la temperatura es un factor que favorece este proceso, ya que con un aumento de esta variable, se produce un incremento en la tasa de reconversión de nutrientes, y por lo tanto un aumento de la productividad del lago. Sin embargo, los valores de los coeficientes de correlación entre la $\mathrm{T}$ y los nutrientes (PT y NT) no fueron relevantes como los encontrados por otros autores en ambientes similares (AMÉ et al., 2002; BAZAN et al., 2005; BONANSEA, 2007).

Por último, la variable Cl-a se correlacionó con el OD $(r=0.51)$ en forma moderada. Este elemento es un requerimiento esencial para el metabolismo de todos los organismos acuáticos que presentan respiración aeróbica y es uno de los gases más importantes en la dinámica y caracterización de los ecosistemas acuáticos. El resto de parámetros analizados no presentaron correlación significativa.

El ACP permitió analizar la interdependencia de variables y encontrar una representación gráfica óptima de la variabilidad de los datos. Con esta técnica se identificaron tendencias generales y relaciones entre las variables: temperatura, $\mathrm{pH}, \mathrm{OD}, \mathrm{DS}, \mathrm{Cl}-\mathrm{a}, \mathrm{NT}$ y PT; las estaciones del año y los sitios de muestreo. Los Componentes Principales (CP) generados resultaron de la combinación lineal de las variables originales previamente estandarizadas. En las Tabla 4 y 5 se muestran los autovalores y autovectores resultantes del ACP sobre la matriz de correlación.

Se utilizaron 2 CP que explicaron el $65,6 \%$ de la variabilidad total de los datos. La CP1 fue la que explicó la mayor proporción de la variabilidad con un $34,6 \%$, 
Tabla 4 - Autovalores del Análisis de Componentes Principales

\begin{tabular}{lccc}
\hline Lambda & Valor & Proporción & Acumulada \\
\hline 1 & 2.421 & 0.346 & 0.346 \\
2 & 2.169 & 0.310 & 0.656 \\
3 & 1.120 & 0.160 & 0.816 \\
4 & 0.640 & 0.091 & 0.907 \\
5 & 0.363 & 0.052 & 0.959 \\
6 & 0.196 & 0.028 & 0.987 \\
7 & 0.091 & 0.013 & 1.000 \\
\hline
\end{tabular}

Tabla 5 - Autovectores del Análisis de Componentes Principales

\begin{tabular}{lrr}
\hline \multicolumn{1}{c}{ Variables } & \multicolumn{1}{c}{ e1 } & \multicolumn{1}{c}{ e2 } \\
\hline $\mathrm{T}$ & 0.52 & 0.14 \\
$\mathrm{pH}$ & -0.45 & -0.22 \\
$\mathrm{OD}$ & 0.01 & 0.61 \\
$\mathrm{DS}$ & $-2.4 \mathrm{E}-04$ & 0.23 \\
$\mathrm{Cl}-\mathrm{a}$ & 0.44 & -0.40 \\
$\mathrm{PT}$ & 0.51 & -0.29 \\
$\mathrm{NT}$ & 0.28 & 0.51 \\
\hline
\end{tabular}

mientras que la $\mathrm{CP} 2$ representó el $31 \%$, concentrando en dos dimensiones la variabilidad estudiada (Tabla 4).

Los autovectores presentado en la Tabla 5 (e1 y e2) mostraron los coeficientes con que cada variable original fue ponderada para conformar las CP1 y CP2. Los valores, en módulo, más altos indican las variables más significativas en cada factor. Se puede visualizar que, al construir la CP1, las variables T, Cl-a y $\mathrm{PT}$ reciben los pesos positivos más altos y la variable $\mathrm{pH}$ el peso negativo más bajo. Para la segunda componente $\mathrm{CP} 2$, las variables OD, NT y Cl-a fueron las más significativas. Las dos primeras tuvieron los pesos positivos más altos, mientras que $\mathrm{Cl}$-a presentó el peso negativo inferior. La ortogonalidad de las CP garantiza que la CP2 provee nueva información sobre variabilidad respecto a la provista por la CP1, es decir explicó variabilidad en la calidad del agua no explicada por la CP1.

Las CP se utilizaron para realizar el gráfico de dispersión (Biplot) (Figura 2), lo que permitió visualizar observaciones y variables en un mismo espacio. Se graficaron las observaciones como rombos, mientras que las variables fueron representadas como vectores desde el origen con terminación en círculos. Los sitios de muestreo se representaron con valores de 1 a 7 ; mientras que las estaciones del año se representaron como V: verano; $\mathrm{O}$ : otoño; I: invierno; P: primavera.

Figura 2 - Biplot. Diagrama de Componentes Principales (CP1-CP2)




Al igual que en la matriz de correlación, por medio del ACP se observó una correlación positiva entre las variables PT, Cl-a y T del agua, asociada a las estaciones primavera y verano, principalmente en los sitios de muestreo 3, 5 y 7 . Además, por este último método, se observó una asociación positiva entre el $\mathrm{NT}$ y el OD con el verano. El pH, con un alto peso en la CP1, estuvo asociado con el invierno y en menor medida con el otoño.

La correlación entre NT y las demás variables fue de baja a nula. Al igual que BONANSEA (2007), se determinó que el $\mathrm{pH}$, con un alto peso en la CP1, no presentó correlación positiva con ninguna variable, pero estuvo asociado con el invierno y en menor medida con el otoño. Por otro lado, se destacó una alta correlación negativa entre $\mathrm{pH}$ y $\mathrm{T}$ del agua.

\section{CONCLUSIONES}

1. En este estudio, a través de variables físicas, químicas y biológicas, se logró establecer una evaluación de la calidad del agua, como también la clasificación trófica del Embalse Río Tercero (Argentina);

2. Durante el periodo analizado el embalse sufrió un cambio de la calidad del agua de mesotrófico a eutrófico, demostrándose su deterioro, lo que evidencia la inminente necesidad de tomar medidas de gestión, tanto en la cuenca como en el reservorio;

3. El análisis multivariado estableció que las variables más significativas para explicar la variabilidad en el reservorio estudiado fueron $\mathrm{Cl}-\mathrm{a}, \mathrm{PT}$ y $\mathrm{T}$, lo que demuestra que los florecimientos algales deberían ocurrir en las estaciones primavera-verano, con menor intensidad en otoño, siendo nulos en invierno;

4. Los resultados obtenidos permiten generar un programa preliminar de monitoreo y gestión extrapolable a otros recursos acuáticos.

\section{REFERENCIAS}

AMÉ, M.; DÍAZ, M.; WUNDERLIN, D. Occurrence of toxic cyanobacterial blooms in San Roque reservoir (Córdoba, Argentina): a field and chemometric study. Environmental Toxicology, v. 18, p. 192-201, 2003.

ANDRADE, E.M. et al. Land use and groundwater quality: The case of Baixo Acaraú, Ceará, Brazil. Revista Ciência Agronômica, v. 41, n. 2, p. 208-215, 2010.

APHA, AWWA, WEF. Standard Methods for the Examination of Water and Wastewater, Ed. Washington, USA, 18th edition, 2000.
BALZARINI M.G. et al. Infostat. Manual del Usuario, Editorial Brujas, Córdoba, Argentina, 2008.

BARROS ALEXANDRE, D.M. et al. The water quality investigation using GIS and multivariable analysis in semiarid regions reservoir. Revista Ciência Agronômica, v. 41, n. 4 , p. $554-561,2010$.

BAZÁN, R. et al. Teledetección y modelado numérico para el análisis de la calidad de agua del embalse Los Molinos. Ingeniería Hidráulica en México, v. 20, n. 2, p. 121-135, 2005.

BOLTOVSKOY, A. M.; FOGGETTA. Limnología física del embalse Río III. Revista Biología Acuática, v. 7, p. 1-26, 1985.

BONANSEA, M. Evaluación de la calidad del agua y estado trófico del embalse Río Tercero, Córdoba, Argentina. Tesis de grado, Universidad Nacional de Río Cuarto, Argentina, 2007.

DI RIENZO J.A., CASANOVES F., BALZARINI M.G., GONZALEZ L., TABLADA M., ROBLEDO C.W. InfoStat versión 2011. Grupo InfoStat, FCA, Universidad Nacional de Córdoba, Argentina. URL http://www.infostat.com.ar.

DO VASCO, N. A. et al. Avaliação espacial e temporal da qualidade da água na sub-bacia do rio Poxim, Sergipe, Brasil. Revista Ambiente \& Água, v. 6, n.1, 2011.

GIRÃO, E.G. et al. Seleção dos indicadores da qualidade de água no Rio Jaibaras pelo emprego da análise da componente principal; Revista Ciência Agronômica, v.38, n.1; p.17-24; 2007.

HELENA, B. et al. Temporal evolution of groundwater composition in an alluvial aquifer (Pisuerga river, Spain) by principal componente analysis. Water research, v. 34, n.3, p. 807-816, 2000.

MANCINI, M. et al. Saprolegniasis en poblaciones silvestres de Astyanax eigenmanniorum y A. fasciatus (PISCES, CHARACIDAE) de Argentina. Revista Brasileira de Medicina Veterinaria, v. 30, n. 2, p. 115-122, 2008.

MANCINI, M. et al. Cianobacterial bloom and animal mass mortality in a reservoir from Central Argentina. Brazilian Journal of Biology, v. 70, p. 841-845, 2010.

MARIAZZI, A.; ROMERO, M. Estimación de la actividad heterotrófica en tres ecosistemas acuáticos con distintos nivel de tropismo. Ecosur, v. 10, p. 61-77, 1983.

MARIAZZI, A. et al. Impact of a nuclear power plant on water quality of Embalse del Río Tercero reservoir (Córdoba, Argentina). Hidrobiología, v. 246, p. 129-140, 1992.

OECD (Organización para la Cooperación y el Desarrollo Económico). Eutrophication of Waters. Monitoring, Assessment and Control. Cooperative Programmers on Monitoring of Inland Waters (Eutrophication Control), Environment Directorate, OECD Paris, Final Report. France. 1982.

PIEROTTO, M. et al. Algas de interés sanitario en embalses del centro-oeste de la provincia de Córdoba-Argentina. Escuela de Salud Publica, v. 11, n. 1, p. 55-63, 2007.

ROCHA, J. L. S. et al. Indicador integrado de qualidade ambiental aplicado à gestão de bacia hidrográfica do rio Junquiriçá, BA, Brasil. Revista Ambiente \& Água, v.5, n.1, p.89-101, 2010. 
RODRÍGUEZ, C. et al. Estudio bacteriológico y químico del embalse Río Tercero (Córdoba). Revista Ciencia Veterinaria, v. 27, p. 3-8, 1997.

RODRÍGUEZ, C. et al. Remote Sensing- GIS to Predict on the Risk of Eutrophication in Aquatic Systems. Space Technologybased tele-health proyect initiatives in Latin America and the Caribbean. United Nations Programme on Space Applications, p. 28-30, 2007.

SILVA, B. A. W.; et al. Gestão ambiental de bacias hidrográficas urbanas. Revista VeraCidade, v.3, n.5, p.1-7, 2006.
RYDING S., RAST W. El control de la eutrofización en lagos y pantanos. Ed. Pirámide. Madrid, España. 1992.

SRIWONGSITANON, N.;SURAKIT, K.;THIANPOPIRUG, $S$. Influence of atmospheric correction and number of sampling points on the accuracy of water clarity assessment using remote sensing application. Journal of Hydrology, v. 401 , p. 203-220, 2011.

STEFOULI, M. et al. E. Monitoring and assessing internal waters (lakes) using operational space born and field measures. European Water Publications 9/10, p. 25-33, 2005. 